\title{
USO DA TOMOGRAFIA DE COERÊNCIA ÓPTICA NA ENDODONTIA: UMA REVISÃO DA LITERATURA
}

\author{
Jussara da Silva Barbosal \\ Ana Priscila Lira de Farias Freitas" \\ Priscilla Kelly Batista Silva Leite ${ }^{\text {III }}$ \\ Luiz Eduardo Marinho Vieiral" \\ Hellen Bandeira de Pontes Santos IV \\ *Fernanda Clotilde Mariz Suassuna
}

\section{RESUMO}

$\mathrm{Na}$ busca do sucesso da terapia endodôntica há uma crescente busca por novas tecnologias, entre elas estão as de obtenção de imagem. Este estudo objetivou analisar na literatura a eficácia e finalidade da Tomografia de Coerência Óptica (OCT) em Endodontia. Trata-se de uma revisão sistematizada da literatura com busca realizada nas bases de dados Pubmed, Scopus e Web of Science. Foram utilizados os seguintes descritores em língua inglesa em conformidade com o Medical Subject Headings (MESH): "Tomography Optical Coherence" e "Endodontics". Os objetivos para a utilização do OCT foram para localização de conduto radicular, localização de fraturas, análise da anatomia interna radicular, detecção de infiltração apical e seu uso como avanço tecnológico. O uso do OCT foi considerado satisfatório in vitro na Endodontia. Conclui-se que há eficácia do OCT na Endodontia in vitro, entretanto mais estudos devem ser realizados e a tecnologia aprimorada para aplicabilidade clínica.

PALAVRAS-CHAVE: Diagnóstico por Imagem. Tomografia de Coerência Óptica. Raiz Dentária. Endodontia.

Mestre em Clínica Odontológica. Faculdade de Enfermagem Nova Esperança - FACENE. Departamento de I Odontologia. CEP: 58067-698, João Pessoa, Paraíba, Brasil.*Autor correspondente: fernandacosta3@hotmail.com ORCID ID: 0000-0001-6123-5266; 0000-0001-5846-288X

Mestre em Clínica Odontológica. Universidade Estadual da Paraíba - UEPB. II Departamento de Odontologia. CEP: 58429-500, Campina Grande, Paraíba, Brasil ORCID ID: 0000-0002-1492-1236; 0000-0001-5701-5164 Mestre em Ciências Odontológicas. Faculdade de Enfermagem Nova Esperança - FACENE. III Departamento de Odontologia. CEP: 58067-698, João Pessoa, Paraíba, Brasil ORCID ID: 0000-0002-6647-6277

Doutora em Ciências Odontológicas. Faculdade de Enfermagem Nova Esperança - FACENE. IV Departamento de Odontologia. CEP: 58067-698, João Pessoa, Paraíba, Brasil ORCID ID: 0000-0002-6596-5220 


\section{INTRODUÇÃO}

A endodontia faz uso de radiografias como parte fundamental do diagnóstico da doença de origem pulpar. Além disso, elas são usadas para determinar a presença de raízes e condutos, reabsorções, cárie, defeitos em restaurações e fraturas radiculares. Muitos pesquisadores tornaram-se conscientes das limitações encontradas nas radiografias dentárias no que se refere a sua característica de bidimensionalidade. ${ }^{1}$ Ultimamente, outros sistemas de diagnóstico por imagem estão sendo sugeridos, a maioria deles utiliza radiação ionizante para a obtenção de imagem, representando assim outro tipo de limitação. ${ }^{2}$

Tomografia de coerência óptica (OCT) é uma tecnologia de imagem para diagnóstico médico que foi introduzido pela primeira vez em 1991 por Huang et al. ${ }^{3}$ Desde então, tornouse uma ferramenta padrão em oftalmologia e um método de imagem promissor para detecção da aterosclerose intracoronária. ${ }^{4,5}$

O OCT é um método não invasivo, não radiativo, constituindo uma ferramenta de diagnóstico baseado em interferómetros ópticos, usando uma fonte de luz de banda larga no infravermelho próximo6, fornecendo detalhes microestruturais da amostra

\section{MATERIAL E MÉTODOS}

Uma revisão sistematizada da literatura foi realizada para a obtenção das produções científicas sobre o uso da Tomografia de Coerência Óptica (OCT) na Endodontia. A busca foi realizada nas bases de dados: Pubmed, Scopus e Web of Science. Utilizaram-se os seguintes descritores em língua inglesa em conformidade com o Medical Subject Headings (MESH): “Tomography, estudada, excelente resolução espacial e é uma das modalidades ópticas de análise mais utilizada. ${ }^{7,8}$

Esta técnica, com princípio semelhante ao do ultrassom, mas usando ondas de luz, é capaz de gerar imagens de secção transversal da microestrutura interna de materiais e sistemas biológicos, com resolução micrométrica. Esta estratégia dá uma vantagem sobre o ultrassom e a ressonância magnética, que não são ideais para imaginologia de tecidos. ${ }^{9}$ Uma variante denominada de Tomografia por Coerência Óptica com fonte de varredura de Frequência (SS-OCT) oferece uma resolução maior do que a radiografia simples ou a tomografia computadorizada (TC) para análise de superfície. ${ }^{10}$

Em endodontia, alguns autores têm referido que o OCT é uma técnica comprovada e confiável para avaliar anatomia intracanal, perfurações radiculares, fraturas de raiz verticais, limpeza do canal após a preparação e, até mesmo, perfurações radiculares., ${ }^{2,11}$ Frente ao exposto, este trabalho tem como objetivo analisar na literatura a eficácia e a finalidade do uso de OCT em Endodontia.

Optical Coherence" e "Endodontics". O operador boleano utilizado foi o "AND". Foram adotados, como critério de inclusão, trabalhos que apresentaram, no resumo, dados relacionados ao uso do OCT na endodontia.

Como critérios de exclusão foram levados em consideração: pesquisas consideradas duplicatas e artigos que fugiram do tema (utilizou outra técnica de imagem e 
trabalhos que associaram a OCT com outra área da odontologia, sem incluir a endodontia). Após a exclusão dos artigos, os estudos escolhidos foram lidos e analisados, sendo classificados segundo o nome do autor, ano de publicação, local de realização do estudo, objetivo da pesquisa e eficácia na utilização do OCT.
Os dados foram analisados descritivamente (distribuições absolutas e percentuais) através do software Microsoft Excel. Foram encontrados 44 artigos, destes 17 se localizaram na base de dados Pubmed, 24 na Scopus e 3 Web of Science, após a aplicação dos critérios de inclusão a amostra final foi constituída por 11 artigos (Figura 1).

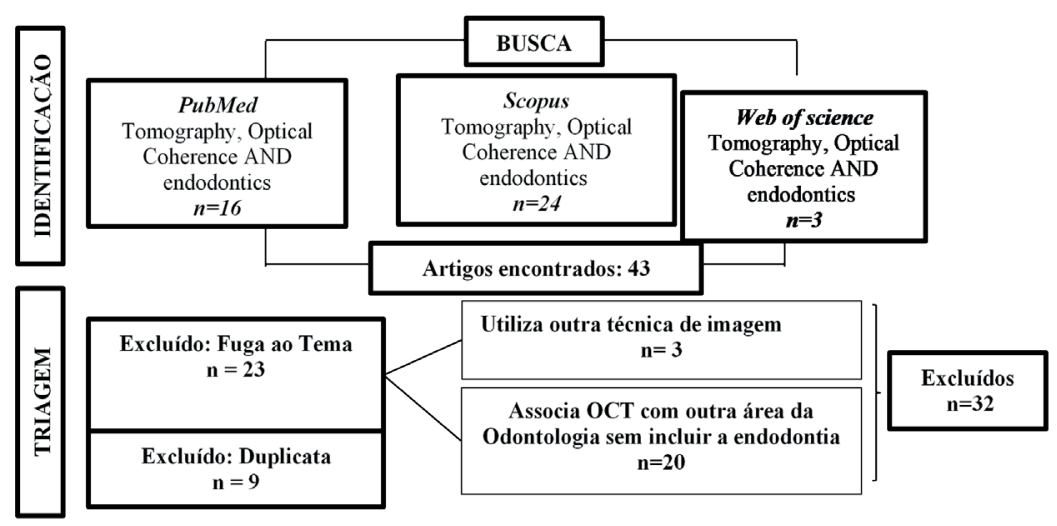

Figura 1: Fluxograma de busca e seleção dos artigos

\section{RESULTADOS E DISCUSSÃO}

Os artigos selecionados tiveram como objetivos a utilização do OCT para localização de conduto radicular, localização de fraturas, análise da anatomia interna radicular, detecção de infiltração apical, estudo do biofilme endodôntico, detecção de lesões perirradiculares e seu uso como avanço tecnológico (Tabela 1).

Segundo Lino et al. ${ }^{12}$, a localização do canal mesio-vestibular possui taxa de detecção in vivo de $33 \%$ em decorrência do acesso e visibilidade limitados, não aumentando o índice com o uso de microscópio. Dessa forma, o SS-OCT foi comparado ao MicroCT (padrão de referência) e a utilização de microscópio dental.
Não houve diferenças significativas entre os três métodos em relação a precisão.

No que diz respeito a capacidade de análise da anatomia radicular interna, no estudo de Shemesh et al. ${ }^{2}$, mostraram uma excelente correlação entre as imagens histológicas e as realizadas em OCT, a partir do uso de aparelho disponível para uso clínico em cateterismo cardíaco, constituído por fio de fibra óptica. Lino et al. ${ }^{12}$ utilizaram o OCT em comparação com a Tomografia Cone-beam para detecção do corno pulpar, tendo o MicroCT como padrão de referência, obtendo como resultado uma correlação positiva entre as técnicas. 
TABELA 1: Distribuição dos artigos segundo Autor, ano de publicação, objetivos e observação da eficácia do OCT.

\begin{tabular}{|c|c|c|c|c|}
\hline Autores & $\begin{array}{l}\text { Ano de } \\
\text { Publicação }\end{array}$ & $\begin{array}{l}\text { Local de } \\
\text { Estudo }\end{array}$ & Objetivos & $\begin{array}{l}\text { Eficácia do } \\
\text { OCT }\end{array}$ \\
\hline Ding et al. & 2019 & China & Detecção de lesões perirradiculares & Sim \\
\hline Suassuna et al. & 2018 & Brasil & $\begin{array}{l}\text { Analisar o selamento apical após obturação } \\
\text { endodôntica }\end{array}$ & Sim \\
\hline Lino et al. & 2015 & Japão & Detectar o corno pulpar & Sim \\
\hline Ebihara et al. & 2015 & Japão & $\begin{array}{l}\text { Observar a superfície ressecada na Apicectomia e } \\
\text { comparar com imagens da região apical }\end{array}$ & Sim \\
\hline Lino et al. & 2014 & Japão & Detectar o segundo canal mésio-vestibular & Sim \\
\hline Yoshioka et al. & 2013 & Japão & $\begin{array}{l}\text { Avaliar a capacidade do OCT na análise de } \\
\text { fraturas radiculares verticais }\end{array}$ & Sim \\
\hline Mortman & 2011 & EUA & Relatar os avanços tecnológicos em endodontia & - \\
\hline Todea et al. & 2010 & Romênia & Relatar os avanços tecnológicos em endodontia & Sim \\
\hline Todea et al. & 2009 & Romênia & Relatar os usos do OCT na Odontologia & - \\
\hline Todea et al. & 2008 & Holanda & $\begin{array}{l}\text { Avaliar a capacidade do OCT na análise de fratu- } \\
\text { ras radiculares verticais }\end{array}$ & Sim \\
\hline Shemesh et al. & 2007 & Holanda & $\begin{array}{l}\text { Avaliar a capacidade do OCT na análise de } \\
\text { fraturas radiculares verticais }\end{array}$ & Sim \\
\hline
\end{tabular}

Em relação a investigação de fraturas, dois artigos discorrem sobre o assunto. Segundo Shemesh et al. ${ }^{11}$, o OCT constitui uma ferramenta poderosa para avaliar a presença de fraturas radiculares verticais, detectando a sua localização específica, sendo possível aumentar o número de informações disponíveis sobre este tipo de fratura com seu uso. No estudo de Yoshioka et al. ${ }^{13}$ a validade e confiabilidade do SS-OCT para detectar a presença de fratura radicular vertical é comparável a utilização de microscópio dental, com traços de fratura, ângulo e linha de desenvolvimento no mesmo local.

Como área crítica na endodontia, a região apical foi estudada com diferentes finalidades: Todea et al. ${ }^{14}$ utilizaram o OCT como ferramenta de comparação entre a infiltração apical, após a utilização de três técnicas de preparo radicular: a técnica convencional e a utilização de dois lasers com comprimentos de onda diferentes (980 nm e 1064 nm), após o preparo químico-mecânico. Em relação ao potencial do OCT, o autor concluiu que este é certamente um método valioso, permitindo uma elevada precisão de avaliação da qualidade do tratamento sem a presença de radiação. Suassuna et al. ${ }^{8}$ analisaram o selamento apical endodôntico promovido por três técnicas de obturação, mostrando o OCT imagens semelhantes com o MicroCT. Já Ding et al. ${ }^{15}$ avaliaram a visualização dos limites de lesões perirradiculares, através de superfícies ósseas intactas.

Em relação a utilização do OCT na Endodontia e na Odontologia, dois artigos relatam este como um avanço tecnológico necessário e ainda promissor. ${ }^{1}$ Todea et al. ${ }^{7}$ ressalta a sua utilização na endodontia para a avaliação da infiltração apical ocorrida em obturações de canais radiculares.

Tomografia de Coerência Óptica (OCT) é relativamente considerada um novo método não-invasivo para a obtenção de imagens com 
potencial em odontologia, de acordo com os artigos selecionados. Esta tecnologia de imagem permite a realização de reconstrução tridimensional (3D) com imagens em tempo real. ${ }^{14,16} \mathrm{Na}$ endodontia, sua atualização mostra ser capaz de impedir iatrogenias, facilitando a localização de condutos pouco comuns e na avaliação intracanal. Além de ser de grande ajuda no diagnóstico de fraturas radiculares por trauma oclusal, perfurações radiculares e avaliação de vazios apicais, após a obturação do canal radicular., ${ }^{8,13}$

$\mathrm{Na}$ odontologia, o OCT pode avaliar a presença de bolhas nas restaurações de resina composta, detectar lacunas na interface dente restauração, falhas na adaptação marginal de restaurações. ${ }^{2,19}$ Além disso, se constitui uma modalidade de diagnóstico promissora para a detecção precoce de cárie oclusal e doenças periodontais. Em tecidos moles, pode ser utilizado para a visualização e segmentação da glândula sublingual, auxiliando no diagnóstico da Síndrome de Sjogren e xerostomia, diagnóstico de lesões vasculares orais e para imagem de lesões orais suspeitas poderia melhorar a precisão do diagnóstico de displasia oral. ${ }^{18}$

A Tomografia Computadorizada Cone-beam (CBCT) é hoje a mais utilizada para formação de imagens tridimensionais, sendo utilizada na detecção de fraturas radiculares por possuir alta resolução. Mas, a presença de restaurações metálicas ou outros materiais metálicos na cavidade oral reduz a sua capacidade de diagnóstico por gerar artefatos. $^{12}$ Portanto, as fraturas estreitas são difíceis de detectar por CBCT na prática clínica. Outra opção de localização de fraturas e condutos consiste na utilização do corante azul de metileno, seguido da observação sob microscópio. No entanto, a dentina não pode ser avaliada em profundidade, sendo difícil distinguir entre uma fratura de variações na morfologia dos condutos. ${ }^{13}$
Outra desvantagem refere-se ao processamento da imagem, por vezes demorado, e com possível interpretação de milhares de imagens. Em contraste, O OCT realiza sua varredura em aproximadamente 15 segundos para uma raiz de $15 \mathrm{~mm} .{ }^{19}$ Além disso, a utilização de uma fibra óptica muito estreita medindo 0,5 mm de diâmetro permite imagens de objetos em alguns micrômetros. Este fio de fibra óptica pode ser implementado de forma independente, ou integrado, diretamente a um cateter terapêutico ou de imagem existentes, podendo assim ser introduzido facilmente em um canal radicular preparado. É flexível, permitindo a penetração através das curvatura., ${ }^{2,19}$

O OCT tem algumas limitações, pouco relatadas na literatura, entre elas está o fato da influência da microestrutura de um tecido específico para a propagação da luz. A dentina é uma estrutura com propriedades ópticas diferente da maioria dos outros tecidos biológicos, isto ocorre, pois os túbulos dentinários constituem uma forma de espalhamento de luz. ${ }^{18}$ No experimento de Shemesh et al. ${ }^{2}$ a dentina radicular foi semitransparente, permitindo gerar imagens do exterior da raiz. No entanto, uma parede de dentina mais espessa não irá permitir penetração suficiente de luz e o contorno exterior da raiz não será visto.

Outro cuidado que deve ser tomado é em relação a refração da luz com índice próximo da água $(1,33)$, se aproxima para os tecidos moles. Tecidos dentários e ósseos possuem índices mais alto de refração $(1,5)$, como consequência, as distâncias dentro do dente são aproximadamente $13 \%$ mais curtas do que o sugerido pelas imagens. Tal limitação pode ser corrigida pelo processamento das imagens ou com a imersão da fibra óptica em um conduto molhado. ${ }^{16}$ Outra desvantagem, do uso na Endodontia, é o custo do cateter do OCT. Uma vez que estes fios são concebidos 
concebidos para uso descartável, por se tratarem de cateteres cardíacos. ${ }^{11}$

Os estudos analisados relatam o uso do OCT in vitro. A partir dos bons resultados expostos é necessário a extrapolação destas pesquisas in vivo, com fim de analisar sua aplicabilidade clínica.

\title{
CONSIDERAÇÕES FINAIS
}

Pede-se comprovar a eficácia do OCT na Endodontia in vitro, sendo esta técnica de imagem utilizada em todas as etapas do tratamento endodôntico, desde a visualização da anatomia até visualização de lesões perirradiculares. Entretanto, a tecnologia ainda está em fase de testes laboratoriais, mais estudos devem ser realizados e a tecnologia aprimorada para aplicabilidade clínica.

\section{USE OF OPTICAL COHERENCE TOMOGRAPHY IN ENDODONTICS: A LITERATURE REVIEW}

\begin{abstract}
In the pursuit of successful endodontic therapy there is a greater search for new technologies, among them are as image search. To analyze in the literature the efficacy and finality of Optical Coherence Tomography (OCT) in endodontics. This was a systematic literature review with a search carried out in Pubmed, Scopus and Web of Science databases. The following descriptors were used in English in accordance with the Medical Subject Headings (MESH): “Tomography, Optical Coherence" and "Endodontics". According to the scientific literature consulted, the objectives for the use of OCT were root canal localization, fracture location, root internal anatomy analysis, detection of apical infiltration and its use as a technological advance. The use of OCT was considered satisfactory in vitro in Endodontics. After reviewing the literature, it is concluded that there is efficacy of the OCT in Endodontics in vitro, however more studies should be performed and the technology improved for clinical applicability.
\end{abstract}

KEYWORDS: Diagnostic Imaging. Tooth Root. Endodontics. 


\section{REFERÊNCIAS}

1. Mortman RE. Technologic advances in Endodontics. Dental Clinics, 2002; 55 (3), 461-80.

2. Shemesh H, Soest GV, Wu MK, Van Der Sluis LWM, Wesselink PR. The Ability of Optical Coherence Tomography to Characterize the Root Canal Walls. J. Endod. 2007; 33 (11), 1369-73.

3. Huang D, Swanson EA, Lin CP, Schuman JS, Stinson WG, Chang W, Hee MR, Flotte T, Gregory K, Puliafito CA, Fugimoto JG. Optical Coherence Tomography. Science. 1991; 254(5035), 1178-1181.

4. Regar E, Van BHM, Van Der GWJ, Serruys PW. Images in cardiovascular medicine. Optical coherence tomography findings at 5-year follow-up after coronary stent implantation. Circulation. 2005; 112 (23). 345-6.

5. Wang Y, Bower BA, Izatt JA, Tan O, Huang D. Retinalblood flow easurement by circumpapillary Fourier domain Doppler optical coherence tomography. J. Biomed. Opt. 2008; 13 (6), 064003-9.

6. Fujimoto, JG. Optical coherence tomography for ultrahigh resolution in vivo imaging. Nat. Biotechnol. 2003; 21 (11), 1361-7.

7. Todea C, Negrutiu ML, Balabuc C, Sinescu C, Topala Fl, Marcauteanu C, Canjau S, Semez G, Podoleanu AG. Optical Coherence Tomography Applications in Dentistry. Timisoara Medical Journal. 2009; 60 (1), 5-17.

8. Suassuna FCM, Maia AMA, Melo DP, Antonino ACD, Gomes ASL, Bento PM. Comparison of microtomography and optical coherence tomography on apical endodontic filling analysis. Dentomaxillofac Radiol. 2018; 47 (2), 20170174.
9. Low AF, Tearney GJ, Bouma BE, Jang IK. Technology insight: optical coherence tomographycurrent status and future development. Nat Clin Pract Cardiovasc Med. 2006: 3 (3), 154-62.

10. Choma MA, Hsu K, Izatt JA. Swept source optical coherence tomography using an allfiber 1300-nm ring laser source. J Biomed Opt, 2005; 10 (4), 044009.

11. Shemesh H, Soest GV, Wu MK, Wesselink PR. Diagnosis of Vertical Root Fractures with Optical Coherence Tomography. Journal of Endodontics. 2008; 34 (6), 739-42.

12. Lino Y, Ebihara A, Yoshioka T, Kawamura J, Watanabe S, Hanada T, Nakano K, Sumi Y, Suda $\mathrm{H}$. Detection of a second mesiobuccal canal in maxillary molars by swept-source optical coherence tomography. Journal of Endodontics. 2014; 40 (11),1865-8.

13. Yoshioka T, Sakaue H, Ishimura H, Ebihara A, Suda H, Sumi Y. Detection of Root Surface Fractures with Swept-Source Optical Coherence Tomography (SS-OCT). Photomedicine and Laser Surgery. 2013; 31 (1), $23-7$.

14. Todea C, Balabuc C, Sinescu C, Filip L, Kerezsi C, Calniceanu M, Negrutiu M, Bradu A, Hughes $M$, Podoleanu AG. En face optical coherence tomography investigation of apical microleakage after laser-assisted endodontic treatment. Lasers Med Sci. 2010; 25 (5), 629-39.

15. Ding J, Liu W, Sadr A, He Y, Ebihara A, Li Y. Detection of simulated periradicular lesions in porcine bone by optical coherence tomography. Journal of endodontics. 2019; 45 (8), 1024-29. 
16. Amaechi BT, Podoleanu AG, Higham SM, Jackson DA. Correlation of Quantitative Light-Induced Fluorescence and Optical Coherence Tomography Applied For Detection and Quantification of Early Dental Caries. J Biomed Opt. 2003; 8 (4), 642-7.

17. Ishibashi K, Ozawa N, Tagami J, Sumi, Y. Swept-source optical coherence tomography as a new tool to evaluate defects of resin-based composite restorations. J. Dent. 2011; 39 (8), 543-548.

18. Jerjes W, Upile T, Conn B , Hamdoon Z, Betz CS, McKenzie G, Radhi H, Vourvachis M, Maay- tah ME, Sandison A, Jay A, Hopper C. In vitro examination of suspicious oral lesions using optical coherence tomography. Br. J. Oral Maxillofac. Surg. 2010; 48 (1), 18-25.

19. Born M, Wolf E. Principles of Optics. Cambridge, UK: Cambridge University

Characterize the Root Canal Walls. Journal of Endodontics. 2007; 33 (11), 1370-3.

20. Kienle A, ForsteR FK, Diebolder R, Hibst R. Light propagation in dentin: influence of microstructure on anisotropy. Phys Med Biol. 2003; 48 (2), 7-14. 